\section{Paola Sarchielli}

Published online: 11 August 2006

\title{
Trigger factors of migraine and tension-type headache
}

Research aimed at verifying precipitating factors for both migraine and tension-type headache attacks has identified several potential triggers with percentages differing widely among studies [1-5]. They include menstrual cycle, particularly in women affected by migraine; environmental factors, especially changes in weather conditions and temperature; afferent stimulation, physical activity and psychological factors, mainly stress or relaxation from stress; sleep disturbances; frequent travelling; oral contraceptives; and finally eating habits, and some food items. According to some authors, lifetime migraineurs experienced headache attacks preceded by triggering factors more frequently than tension-type headache sufferers [5]. Moreover, certain endogenous and exogenous factors seem to differentiate migraine from tension-type headache and it is of interest to note in this regard that three of the precipitating factors considered more migraine-specific (i.e., weather, smell and smoke) involve the nose/sinus system, suggesting a greater significance of this system in migraine than is generally believed [4].

The aim of the Wöber et al. [6] study was to investigate the consistency of the recurrence of precipitating factors in migraineurs and tension-type headache patients enrolled from a clinical outpatient setting and from a sample, although not conspicuous, of the general population. The authors found that the majority of trigger factors precipitate migraine and tension-type headache only occasionally and not consistently in almost the majority of cases. In contrast to previous experiences, no differences emerged in this study in the percentages of factors referred as attack precipitants between the two patient groups.

The occasional occurrence of some precipitating factors in some but not all migraine and tension-type headache attacks supports the view that they can surely play a role, although not exclusively, in influencing the pathophysiological substrate underlying the threshold for attacks in both headache forms. This predisposed substrate can be, from time to time, influenced by different inner and outer stimuli. Mechanisms involved in the potentiality of different factors in precipitating attacks by activating strategic brain areas involved in the generation and abnormal processing of painful information from the head remain to be established. New imaging techniques and particular experimental settings could be helpful in clarifying this important issue, at least for some attack precipitants, as recent experiences have found [7]. 
The non-consistency of the recurrence of some precipitating factors among attacks in the same patients should prompt the design of longitudinal prospective studies, which overcome the difficulty of patients responding adequately with the aid of a questionnaire based only on recall of their previous attacks (easier to remember the most recent attacks), like the one used by the authors.

Another relevant feature of the Wöber et al. [6] study is the comparative assessment between personal experience and theoretical knowledge of patients. Thus, they could verify the overestimation of some factors as potential triggers for attacks, particularly in migraineurs examined in the clinical settings. The latter have more frequent and disabling attacks and are more motivated to observe potential trigger factors more closely.

Deceptive information emerged, in particular on the role played by weather, and some foods such as cheese and chocolate, for which data are inconsistent or lacking, as well as for oral contraceptives, as precipitants of attacks.

Despite false preconceptions, theoretical knowledge seemed not to have influenced the personal experience of patients because the number of patients having heard or read of certain factors as attack precipitants was lower than that of patients who had experienced the same factors directly.

The lack of clear and correct information for patients, even those attending a clinically specialised centre, strongly emphasises the importance of patient education with the aim of furnishing a clear description of the known trigger factors based on the available scientific evidence. This should be the first step to successively identify, by means of headache diaries, specific precipitants eventually involved in the attacks of the single patient, even if the precipitants are not consistent but at least more frequent in triggering attacks. After their identification, physicians should provide strategies to remove precipitants that can be avoided or modified.

\section{P. Sarchielli, MD (两) Headache Centre Neurological Clinic,} Department of Medical and Surgical Specialties and Public Health University of Perugia, Italy e-mail: headache@unipg.it Tel.: +39-075-578-3609 Fax: +39-075-578-3609

\section{References}

1. Rasmussen BK (1993) Migraine and tension-type headache in a general population: precipitating factors, female hormones, sleep pattern and relation to lifestyle. Pain 53:65-72

2. Robbins L (1994) Precipitating factors in migraine: a retrospective review of 494 patients. Headache 34:214-216

3. Turner LC, Molgaard CA, Gardner CH, Rothrock JF, Stang PE (1995) Migraine trigger factors in a non-clinical Mexican-American population in San Diego county: implications for etiology. Cephalalgia 15:523-530
4. Spierings EL, Ranke AH, Honkoop PC (2001) Precipitating and aggravating factors of migraine versus tension-type headache. Headache 41:554-558

5. Zivadinov R, Willheim K, SepicGrahovac D, Jurjevic A, Bucuk M, Brnabic-Razmilic O, Relja G, Zorzon M (2003) Migraine and tension-type headache in Croatia: a populationbased survey of precipitating factors. Cephalalgia 23:336-343
6. Wöber C, Holzhammer J, Zeitlhofer J, Wessely P, Wöber-Bingöl C (2006) Trigger factors of migraine and tension-type headache: experience and knowledge of the patients. J Headache Pain 7:188-195

7. Siniatchkin M, Averkina N, Gerber WD (2006) Relationship between precipitating agents and neurophysiological abnormalities in migraine. Cephalalgia 26:457-465 